\title{
Uso de podcast para apoio a aprendizagem de algoritmos em curso de graduação em Computação
}

\author{
Caique Ferreira, João Marcos Soares Anjos, João Vinícius Ottoni, \\ Milton P. de Almeida Castro, Valguima V. V. A. Odakura, \\ Rodrigo P. da Silva Sacchi, Carla A. Barvinski \\ ${ }^{1}$ Faculdade de Ciências Exatas e Tecnologia (FACET) \\ Universidade Federal da Grande Dourados (UFGD) \\ Dourados-MS-Brasil \\ \{caiquef.ms, joaomarcoscomp, ottonijehad, castro.milton07, \\ valguima.odakura, rodrigosacchi.ufgd, carlabarvinski\}@gmail.com
}

\begin{abstract}
This article explores the development of a set of podcast episodes on algorithms, called Algorithm Podcast, which is a teaching material whose purpose is to serve as a supplementary material to this discipline. The series produced was presented to a group of Computing graduate students for a semester. Although few students know podcasts at the beginning of the semester, after listening the serie, most of them evaluated that podcasts are useful for learning algorithms.
\end{abstract}

Resumo. Este artigo discorre sobre o desenvolvimento de um conjunto de episódios de podcasts sobre algoritmos, denominado Algoritmo Podcast, que é um material didático cuja finalidade é servir de material suplementar a essa disciplina. A série produzida foi apresentada para uma turma de estudantes de graduação em Computação durante um semestre. Apesar de poucos estudantes conhecerem podcasts no início do semestre, após ouvir a série, a maioria deles avaliou que os podcasts são úteis para aprendizagem de algoritmos.

\section{Introdução}

Dentre as várias tecnologias introduzidas pela Web e Tecnologias da Informação e Comunicação (TIC) está o podcast. Podcast é caracterizado como sendo gravações de áudio ou vídeo digital que, associado ao serviço de Podcasting (Portable On Demand Broadcasting), disponibiliza recurso automático de divulgação e distribuição de episódios de podcast. Podcasting é uma forma de publicação de conteúdos dessas mídias na Web na forma de seriado composto de episódios abordando um tema específico. O conteúdo de um podcast pode ser acessado diretamente, ou ainda transferido diretamente para o dispositivo do usuário, seja esse um desktop ou dispositivo móvel.

Podcasts podem ser úteis para fins educacionais, podendo o seu uso ser classificado, segundo [Deal 2007], em três categorias: gravação de aulas nas mídias de vídeo ou áudio para revisão; como conteúdo suplementar de material didático; além de podcasts produzidos por alunos. Segundo [Freire 2013] o podcast potencializa ações pedagógicas que possuem caráter prático, ajudando com possibilidade do reaproveitamento de materiais já criados, enriquecendo debates, apresentando de forma atrativa conteúdos teóricos, 
e facilitando a transmissão de informação. Mais ainda, [Lazzari 2009] afirma que o uso de podcasts no ensino superior é uma oportunidade em potencial e que seu uso melhora o desempenho dos alunos, promove a elaboração cognitiva e aumenta a sua crítica de pensamento.

Cursos de graduação em Computação tem como base disciplinas que envolvem o uso de Algoritmos para a formação do estudante. Essas disciplinas, na sua parte introdutória, utilizam uma linguagem natural, o Português estruturado, também conhecido como portugol. Entre os conceitos abordados, destacam-se: estruturas sequenciais, condicionais e de repetição, arranjos e procedimentos e funções.

Nesse sentido, neste artigo é relatada a produção e o uso de podcasts para aprendizagem de Algoritmos. A série produzida foi denominada Algoritmo Podcast, e foi concebida para ser utilizada como material didático suplementar para apoiar as disciplinas básicas de Algoritmos em cursos de graduação em Computação. Os resultados do uso da série com uma turma iniciante de graduação mostram que, apesar de poucos estudantes conhecerem os podcasts no início do semestre, ao final do mesmos eles perceberam a sérieAlgoritmo Podcast como útil para sua aprendizagem de Algoritmos.

Este artigo está organizado da seguinte forma. Na seção 2 são descritos os trabalhos relacionados, que utilizam podcast na educação. Na seção 3 é apresentado o desenvolvimento da série Algoritmo Podcast, seguida da seção 4, em que são discutidos os resultados obtidos da avaliação de estudantes que ouviram a série enquanto cursavam uma disciplina de Algoritmos. Por fim, na seção 5, as considerações finais são tecidas.

\section{Trabalhos relacionados}

Diversas pesquisas têm focado no uso de podcasts como ferramenta para auxiliar o ensino-aprendizagem no ensino superior, na forma de uso suplementar. Contudo, esses trabalhos mostram uma possibilidade educacional a ser explorada, como o uso de podcasts para a aprendizagem de Algoritmos.

[Evans 2008] apresentou um estudo sobre o uso de podcast por estudantes do ensino superior. No estudo os alunos utilizaram podcasts com conteúdos de revisão de uma disciplina e foram questionados sobre seus benefícios. Os estudantes perceberam os podcasts como ferramentas mais eficientes para revisão de conteúdos do que livros textos. Além disso, os estudantes relataram que são mais receptivos ao material didático na forma de podcast do que as aulas tradicionais ou livros textos. Nesse contexto, o uso de podcast para adultos em ensino superior aparece como ferramenta promissora.

[Cunha et al. 2009] descrevem a utilização de podcast na graduação, em que propuseram aos estudantes transpor o material escrito de seus trabalhos para a forma de podcast de áudio ou vídeo. Com base na análise das experiências e opiniões dos alunos foi concluído que apesar das avaliações positivas, o uso de podcast não sobrepõe o formato de texto escrito. Diversos alunos relataram que a informação apresentada na forma de texto é assimilada mais facilmente, contudo a maioria dos estudantes se mostrou receptiva aos podcasts de áudio como complementos dos conteúdos escritos. Desse modo, mostra-se promissor o uso de podcasts complementando os materias tradicionais.

[Kurtz et al. 2007] utilizaram podcasts em vídeo com estudantes da disciplina de Engenharia de Software de um curso de graduação em Ciência da Computação. Os au- 
tores produziram podcasts correspondentes ao conteúdo da disciplina e trabalharam na forma de sala de aula invertida, ou seja, os estudantes assistiam os podcasts antes das aulas e resolviam problemas durante as aulas. Essa abordagem permitiu que o tempo de aula fosse dedicado a sessões de resolução de problemas e projetos. Analisando o aproveitamento, detectou-se que os estudantes que receberam aulas em podcast produziram projetos melhores do que a turma anterior da disciplina, a qual teve aulas convencionais.

Segundo [McGarr 2009], o uso de podcast no ensino superior resume-se a três modalidades: o mais comum é o de substituição, em que se usam gravações de aulas passadas com o propósito de revisão de conteúdos; o segundo mais encontrado é o de uso suplementar, como material adicional; o terceiro, o menos comum, é o do uso criativo, em que os próprios estudantes produzem seus podcasts. Esse trabalho descreve que na substituição os estudantes tornam-se receptores passivos da informação, enquanto que no suplementar, os estudantes podem se aprofundar nos conteúdos e explorar novos pontos de vista e, por fim, o uso criativo é o que permite que os estudantes se tornem criadores de conhecimento ao invés de meros receptores. Como resultado, esse trabalho conclui que o uso de podcast e sua influência nas aulas tradicionais não deve ser determinado pela tecnologia em si, mas pela forma que é percebida pela instituição, seus docentes e alunos, sendo fortemente influenciado pela pedagogia dominante empregada.

\section{Metodologia de desenvolvimento}

Algoritmos como disciplina é um dos alicerces da computação. Contudo, essa é uma disciplina que possui alto nível de evasão e reprovação. Buscando apoiar o processo de ensino e aprendizagem para essa disciplina, foi produzida uma série de podcasts sobre assuntos introdutórios de Algoritmos, denominada Algoritmo Podcast.

A série Algoritmo Podcast foi desenvolvida com o objetivo de servir como material suplementar para disciplinas básicas sobre Algoritmos. O uso de podcast como material suplementar é relatado por [McGarr 2009] e [Fernandez et al. 2009], que ressaltam que seu uso possibilita a estudantes o aprofundamento nos conteúdos, o aumento na motivação, a aproximação com o professor, o desenvolvimento de habilidades e a exploração de novos pontos de vista. Para esta série, estabeleceu-se que os podcasts apresentariam os conteúdos com os conceitos iniciais, fornecendo exemplos do cotidiano dos estudantes.

O desenvolvimento da série Algoritmo Podcast envolveu 3 professores das disciplinas básicas de Algoritmos e Estruturas de Dados, que elaboraram os roteiros para gravação, além de 4 acadêmicos que realizaram as gravações, edições e distribuição dos áudios.

A metodologia de desenvolvimento dos episódios de podcast adotou um processo composto de três fases subsequentes, cada qual envolvendo atividades específicas [Deal 2007]. As fases do processo foram: a) produção de podcasts; b) publicação de podcasts; c) entrega e reprodução.

A produção de podcasts consiste na escolha de temas, de formatos para publicação, equipamentos e recursos. Seguindo as orientações de [Deal 2007], a definição de temas incluiu o desenvolvimento de roteiro abordando cada episódio. Esse processo 
V Congresso Brasileiro de Informática na Educação (CBIE 2016)

Anais dos Workshops do V Congresso Brasileiro de Informática na Educação (CBIE 2016)

envolve o planejamento, a redação do conteúdo, a gravação de áudio, bem como sua edição e compactação de arquivos.

As atividades de produção de podcasts possuem requisitos específicos que envolvem o uso de hardware para gravação, como microfones e câmeras digitais e software para edição e compressão de áudio e/ou segmentos de vídeo. Seu conjunto de atividades é o mais demorado e delicado do processo, pois envolve também o planejamento e a escrita de conteúdo, além da necessidade de gerar feeds RSS (Really Simple Syndication) [Deal 2007]. Esse feed é um arquivo XML (eXtended Markup Language) que lista a localização dos episódios de podcasts. Esse tipo de arquivo armazena informações como título, data de publicação, descrição do podcast, entre outras.

A fase de publicação de podcast envolve o envio do arquivo de áudio e o feed RSS para um servidor Web, para notificação do público sobre a existência do podcast, divulgando a localização do feed RSS. Quando um podcast é publicado como uma notificação RSS feed, os assinantes desses feeds são notificados automaticamente por agregadores RSS quando um novo material é disponibilizado. Dessa forma, o acompanhamento da série pode ser automatizado através desses agregadores de podcast, tal como o iTunes ou o Google Play Music, que periodicamente verificam os feeds, os atualiza e obtém automaticamente episódios novos desde a última verificação. Além dos agregadores, os podcasters costumam publicar seus podcasts inserindo links em blogs ou Web sites.

A última fase do processo de desenvolvimento de podcasts foi a entrega e reprodução. Essa fase é caracterizada pela adição de um novo feed RSS pelo ouvinte e o download de todos os episódios referenciados nesse feed RSS pelo agregador. Permitindo que os ouvintes acessassem os podcasts diretamente de seus computadores pessoais ou através de seus dispositivos móveis, tais como, tocadores de MP3/Vídeo, celulares e tablets.

Seguindo a metodologia apresentada, para o processo de desenvolvimento da série, o conteúdo da disciplina de Algoritmos foi dividido em 10 episódios, com a distribuição ilustrada na Tabela 1. Para cada um dos episódios foi elaborado um roteiro, que posteriormente orientou a narração e gravação de áudio.

Table 1. Estrutura da série Algoritmo Podcast.

\begin{tabular}{|l|l|}
\hline Episódio & Conteúdo \\
\hline \hline 1 & Conceito de algoritmos \\
\hline 2 & Tipos de dados e Operadores \\
\hline 3 & Variáveis, constantes e estrutura sequencial \\
\hline 4 & Estrutura condicional - se, se-senão \\
\hline 5 & Estruturas de repetição - para..faça \\
\hline 6 & Estruturas de repetição - enquanto..faça \\
\hline 7 & Estruturas de repetição - faça..enquanto \\
\hline 8 & Vetores \\
\hline 9 & Matrizes \\
\hline 10 & Procedimentos e funções \\
\hline
\end{tabular}

Os roteiros foram preparados priorizando uma linguagem informal, com exemplos do cotidiano dos estudantes de graduação. Os assuntos foram abordados de maneira in- 
V Congresso Brasileiro de Informática na Educação (CBIE 2016)

Anais dos Workshops do V Congresso Brasileiro de Informática na Educação (CBIE 2016)

trodutória. O tempo de duração dos episódios criados ficou em torno de 5 minutos e essa característica é baseada nas conclusões de [Deal 2007], que relatam uma pesquisa entre estudantes, apontando que a duração razoável para um podcast deve estar entre 6 e 10 minutos. Além disso, escolheu-se uma música para abertura e finalização dos episódios, o que fornece uma identidade musical para a série. A gravação contou com a participação das vozes de 4 estudantes, que dialogavam sobre o conteúdo durante os episódios.

\section{Experimentos e Resultados}

A série Algoritmos Podcast produzida foi apresentada para uma turma iniciante de graduação no curso de Sistemas de Informação, especificamente na disciplina de Algoritmos e Estruturas de Dados I. Foi aplicado um questionário inicial aos 49 estudantes a fim de investigar o conhecimento prévio deles em relação ao uso de podcasts.

Em relação ao conhecimento prévio da tecnologia podcast, $18.4 \%$ responderam que já conheciam essa tecnologia antes, $49 \%$ responderam que conheciam mais ou menos e o restante, $32.7 \%$, responderam que não sabiam o que era um podcast. Ainda, desse total de estudantes, quando foram questionados sobre a utilização de podcast no seu cotidiano, a maioria deles, $84.1 \%$, disse não utilizá-los, enquanto que $15.9 \%$ relatou utilizá-los. Estes dados indicam que, mesmo sendo alunos de graduação em curso de Computação, os estudantes não estão familiarizados com a tecnologia podcast.

Os estudantes que utilizam podcasts no seu cotidiano foram indagados sobre os temas de suas preferências ao ouvir os podcasts. A Figura 1 ilustra os resultados apresentados, apontando que os dois assuntos de maior audiência são o entretenimento e o aprendizado tecnológico, ambos com 40\%, seguidos de notícias com $13.3 \%$ e estudo de idiomas com $6.7 \%$.

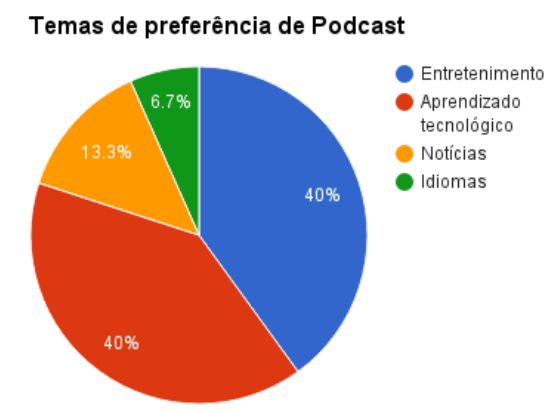

Figure 1. Temas de preferência de podcast.

\subsection{No início da série}

A série Algoritmo Podcast foi apresentada em sala de aula para os 49 estudantes. Neste momento eles ouviram o primeiro episódio da série, que contém conceitos sobre Algoritmos. Após ouvi-lo, os estudantes responderam sobre a utilidade dos podcasts para aprendizagem do tema. Utilizando a escala Likert, a maioria dos alunos, 70.8\%, concorda que ouvir podcasts tem utilidade para o ensino e aprendizagem, enquanto que $16.7 \%$ concorda totalmente, seguidos de $10.4 \%$ que são indiferentes e $2 \%$ que discordam. Na Figura 
V Congresso Brasileiro de Informática na Educação (CBIE 2016)

Anais dos Workshops do V Congresso Brasileiro de Informática na Educação (CBIE 2016)

2 tem ilustrado esses resultados sobre a utilidade dos podcasts para aprendizagem de Algoritmos.

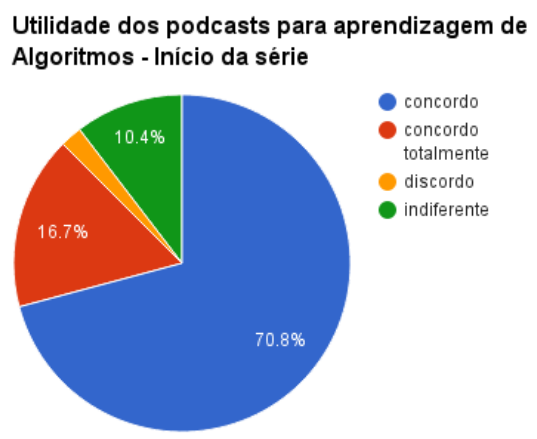

Figure 2. Utilidade dos podcasts para aprendizagem de Algoritmos - no início da série.

Nessa parte do questionário os estudantes também foram perguntados sobre o melhor período para ouvir os podcasts. Antes e depois da aula foram os momentos mais destacados, com $38.3 \%$ e $31.7 \%$, respectivamente, seguidos de antes da prova por $18.3 \%$, não sei por $8.3 \%$ e durante a aula por $3.3 \%$ dos estudantes. A Figura 3 apresenta os valores sobre o momento de preferência para ouvir podcasts.

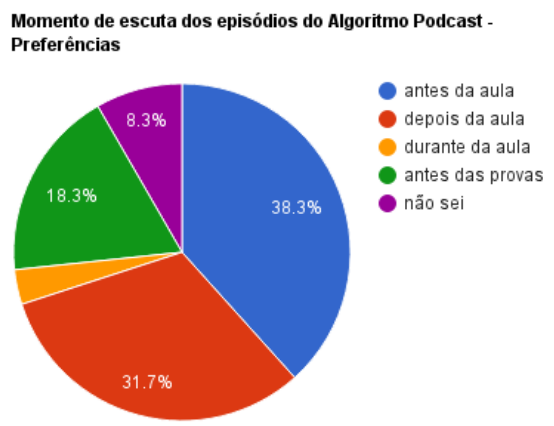

Figure 3. Momento de preferência para escutar podcasts sobre Algoritmos.

\subsection{Ao final da série}

Durante o curso da disciplina, foram indicados novos episódios, correspondentes aos conteúdos abordados na aula seguinte, para que os alunos pudessem ouvir antes da aula. Após o período, foi aplicado um questionário para saber sobre a percepção dos estudantes em relação a série ouvida. Desta etapa participaram 41 estudantes, pois 8 estudantes evadiram antes do término da disciplina, ou seja, aproximadamente $16 \%$.

Os estudantes foram perguntados se escutar a série Algoritmos Podcast os motivou a estudar mais a disciplina de Algoritmos. Os resultados obtidos conforme ilustra a Figura 4 mostram que $15 \%$ concordaram totalmente que sua motivação para os estudos 
V Congresso Brasileiro de Informática na Educação (CBIE 2016)

Anais dos Workshops do V Congresso Brasileiro de Informática na Educação (CBIE 2016)

da disciplina de Algoritmos aumentou, 57,5\% dos alunos concordaram que sua motivação para os estudos também melhorou, enquanto que 22,5\% declararam-se indiferentes e 5\% discordaram desse aspecto.

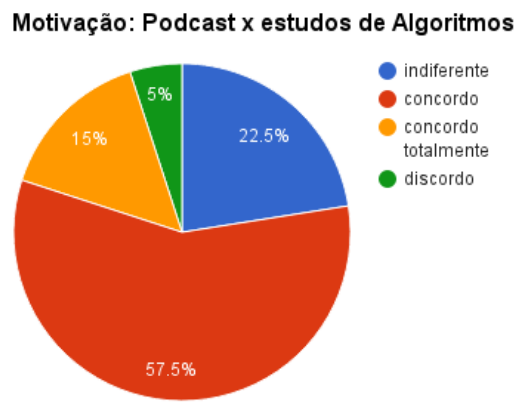

Figure 4. Motivação para estudar Algoritmos após ouvir os episódios da série Algoritmos Podcast.

Perguntados sobre em quais momentos ouviram os episódios da série Algoritmos Podcast, $54.3 \%$ dos alunos declararam que os escutaram em casa ou no trabalho, quando estavam estudando conteúdo da disciplina, outros $37 \%$ prefereriram escutar antes da aula, no trajeto para a universidade, enquanto 4,3\% optaram por ouvir depois da aula ou antes da prova, conforme ilustrado na Figura 5.

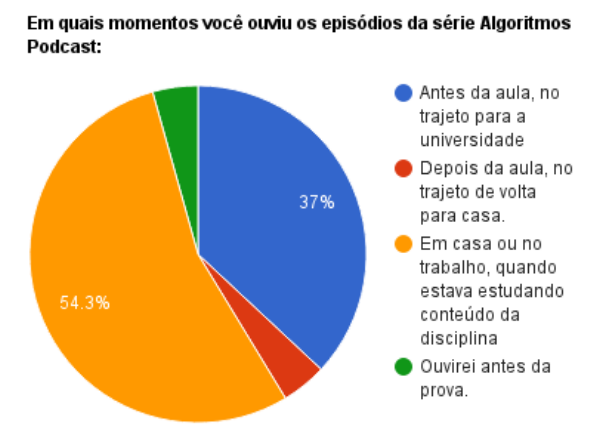

Figure 5. Momentos em que os alunos ouviram os episódios da série Algoritmos Podcast.

Os estudantes também foram questionados se ouvir os podcasts havia prendido sua atenção. A maioria, $57.5 \%$ e $10 \%$, concordaram e concordaram totalmente, respectivamente. Apenas $7.5 \%$ discordaram e $25 \%$ foram indiferentes. Esses resultados são ilustrados na Figura 6.

Os estudantes foram perguntados se os conteúdos da série Algoritmos Podcast foram apresentados de forma clara. A maioria dos estudantes, $70 \%$, concordaram e $22.5 \%$ concordaram totalmente, enquanto que os demais estudantes empatam em $2.4 \%$ para indiferente, discordo e discordo totalmente, conforme os dados ilustrados na Figura 7. 
V Congresso Brasileiro de Informática na Educação (CBIE 2016)

Anais dos Workshops do V Congresso Brasileiro de Informática na Educação (CBIE 2016)

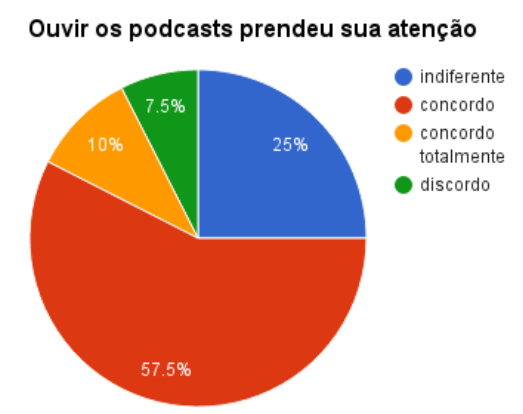

Figure 6. Atenção dos estudantes ao ouvir os podcasts.

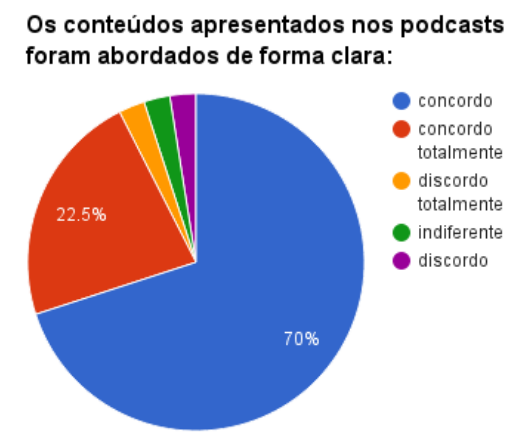

Figure 7. Percepção dos estudantes sobre a abordagem dos conteúdos da série Algoritmos Podcast.

Quando perguntados sobre a adequação do tempo de duração dos episódios, a maioria concordou que o tempo de aproximadamente 5 minutos é adequada, sendo que $47.5 \%$ concordaram e $17.5 \%$ concordaram totalmente. Apenas $2.4 \%$ discordaram e $17.5 \%$ foram indiferentes. Esses dados são mostrados na Figura 8. O tempo de duração avaliados pelos estudantes está de acordo com [Deal 2007], que sugere um episódio tenha entre 6 e 10 minutos.

Os estudantes foram também questionados sobre a utilidade dos podcasts da série Algoritmos Podcast. Dos estudantes participantes, $48.8 \%$ responderam concordo e $39 \%$ concordo totalmente, enquanto $7.3 \%$ responderam indiferente e $2.4 \%$ responderam tanto discordo como discordo totalmente. Os resultados podem ser visualizados na Figura 9. Ao final da série, os estudantes alteraram sua percepção sobre a utilidade da série, como pode ser visualizado ao observar as Figuras 2 e 9 . No início da série a utilidade foi avaliada com $87.5 \%(70.8 \%+16.7 \%)$ entre concordo totalmente e concordo, enquanto que no final da série, a utilidade foi avaliada com $87.8 \%(48.8 \%+39 \%)$ entre concordo totalmente e concordo.

Para mostrar as experiências adquiridas pelos alunos ao ouvir os episódios da série Algoritmos Podcast, foi elaborada uma sumarização das respostas dos dois ques- 
V Congresso Brasileiro de Informática na Educação (CBIE 2016)

Anais dos Workshops do V Congresso Brasileiro de Informática na Educação (CBIE 2016)

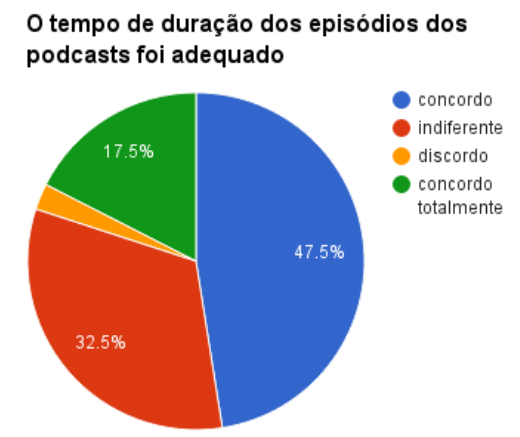

Figure 8. Tempo de duração dos episódios da série Algoritmo Podcast.

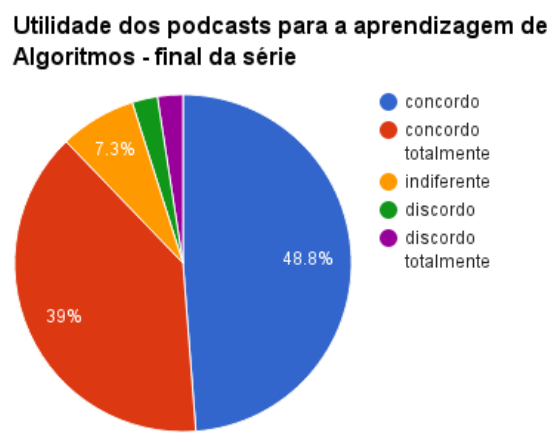

Figure 9. Utilidade dos podcasts para aprendizagem de Algoritmos - final da série.

tionários em forma de nuvens de palavras. A Figura 10(a) tem apresentada a sumarização das respostas do primeiro questionário, enquanto que na Figura 10(b) tem a sumarização das respostas do segundo questionário. Na primeira, destacam-se as palavras: bom, interessante, conhecimento, além de agregar, aprender, ajudar, entre outras. Na segunda, destacam-se as palavras: conteúdo, além de ajudar, esclarecer, entender, compreender, interessante e gostar. As nuvens de palavras mostram que os estudantes tiveram percepções positivas sobre a série ao incluir palavras como entender, compreender e esclarecer.

A produção e distribuição da série Algoritmo Podcast para estudantes do curso de graduação em Sistemas de Informação mostrou-se uma experiência educacional promissora. A utilização da tecnologia podcast aliada a aprendizagem de Algoritmos despertou interesse dos estudantes pelo tema, aproveitou-se da tecnologia móvel por permitir que os estudantes ouvissem a série onde e quando quisessem, em especial no seu trajeto para a universidade. Além disso, o resultado mais importante foi a percepção dos estudantes de que ouvir a série é útil para a aprendizagem de Algoritmos.

\section{Considerações Finais}

Neste artigo foi apresentado o processo de desenvolvimento da série Algoritmos Podcast, composta por 10 episódios abordando conteúdo básico sobre Algoritmos. A série foi 


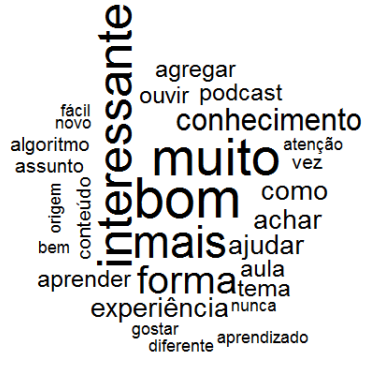

(a) Após ouvir o primeiro episódio da série Algoritmo Podcast.

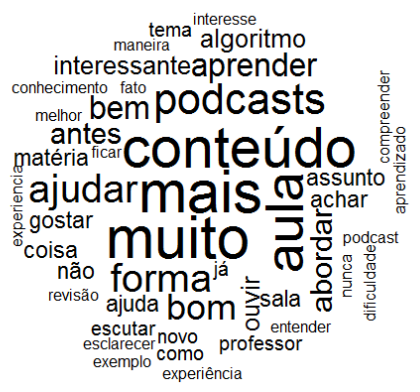

(b) Após ouvir toda a série Algoritmo Podcast.

\section{Figure 10. Nuvens de palavras formadas após ouvir os episódios da série Algo- ritmo Podcast.}

desenvolvida com o objetivo de servir de material suplementar para as disciplinas iniciais que tratam de Algoritmos. A série foi avaliada por uma turma da disciplina de Algoritmos e Estrutura de Dados 1, de um curso de graduação em Computação, durante um semestre letivo e foi bem recebida pelos alunos, que consideraram que os podcasts podem aumentar a motivação para os estudos, bem como ajudar a compreender o conteúdo.

Como trabalhos futuros, devemos considerar que outras séries de podcasts podem ser criadas sobre Algoritmos ou ainda considerar a possibilidade da criação de séries com outros conteúdos de Computação, uma vez que existem poucas séries educacionais sobre o tema disponíveis. Além disso, deve-se trabalhar na divulgação da série produzida, para que possa ser utilizada por outras turmas de graduação em Computação, alcançando mais estudantes do que a turma piloto descrita nesse artigo.

\section{References}

Cunha, C., Teixeira, S., Maciel, R., and Aguiar, C. (2009). Aprender com podcasts. In Actas do X Congresso Internacional Galego-Português de Psicopedagogia. Braga: Universidade do Minho, pages 5493-5498.

Deal, A. (2007). Podcasting. Teaching with Technology.

Evans, C. (2008). The effectiveness of m-learning in the form of podcast revision lectures in higher education. Computers \& education, 50(2):491-498.

Fernandez, V., Simo, P., and Sallan, J. M. (2009). Podcasting: A new technological tool to facilitate good practice in higher education. Computers \& Education, 53(2):385-392.

Freire, E. P. A. (2013). Podcast: novas vozes no diálogo educativo. Interacções, 9(23).

Kurtz, B. L., Fenwick Jr, J. B., and Ellsworth, C. C. (2007). Using podcasts and tablet pcs in computer science. In Proceedings of the 45th annual southeast regional conference, pages 484-489. ACM.

Lazzari, M. (2009). Creative use of podcasting in higher education and its effect on competitive agency. Computers \& Education, 52(1):27-34.

McGarr, O. (2009). A review of podcasting in higher education: Its influence on the traditional lecture. Australasian Journal of Educational Technology, 25(3). 